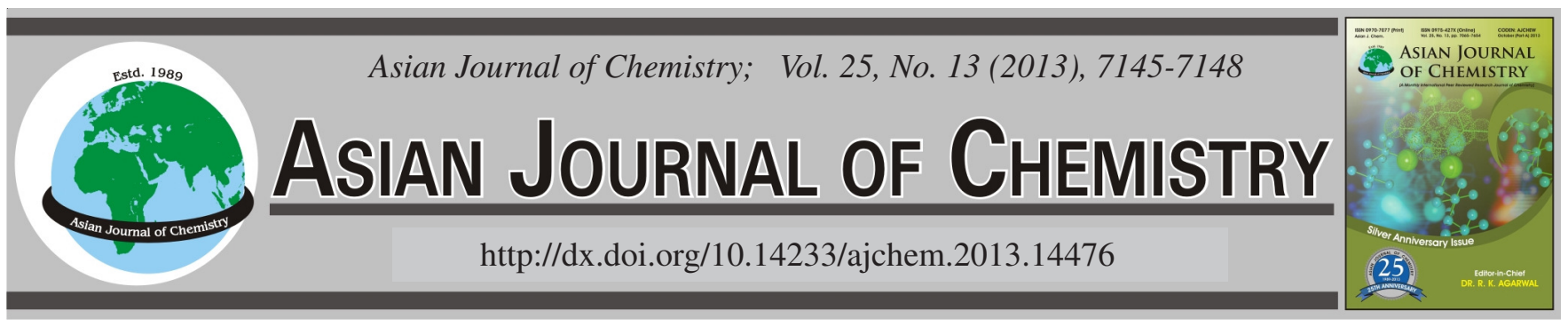

\title{
Synthesis, Characterization and Antibacterial Properties of Novel One-Dimensional Composite Nanofibers
}

\author{
LikUn ZhANG ${ }^{1}$, ChUNPING $\mathrm{LI}^{2, *}$, XUAN ZhANG ${ }^{1}$ and ShAN WANG ${ }^{2}$
}

${ }^{1}$ Inner Mongolia Vocational College of Chemical Engineering, Huhhote 010070, P.R. China

${ }^{2}$ Chemical Engineering College, Inner Mongolia University of Technology, Huhhote 010051, P.R. China

*Corresponding author: Tel./Fax: +86 471 6500910; E-mail: zhanglkhx@126.com

(Received: 25 August 2012;

Accepted: 14 June 2013)

AJC-13659

\begin{abstract}
The novel $\beta$-cyclodextrin/poly(N-vinylpyrrolidone) ( $\beta$-CD/PVP) composite nanofibers containning silver nanoparticles were successfully prepared via electrospinning technique. Silver nanoparticles were synthesized using silver nitrate as the precursor, ethanol as solvent and poly(N-vinylpyrrolidone) (PVP) introduced as a capping agent and the Ag nanoparticles were obtained in solution. The colloidal silver prepared in a PVP solution was directly mixed into $\beta$-CD/DMF solution matrix to form the composite solution and then the prepared solution was electrospun to fabricate AgNPs/ $\beta$-CD-PVP composite nanofibers. Scanning electron microscopy was used to study the morphology of the composite fibers, transmission electron microscopy images showed that the silver nanoparticles were well separately dispersed in $\beta-\mathrm{CD} / \mathrm{PVP}$ matrix. The antimicrobial properties were also investigated.
\end{abstract}

Key Words: Nanofibers, Electrospining, Silver nanoparticles, $\beta$-Cyclodextrin, Poly(N-vinylpyrrolidone).

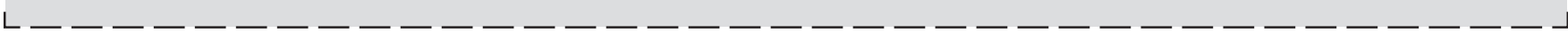

\section{INTRODUCTION}

Organic-inorganic hybrid nanostructures have attracted increasing attention in the fabrication of various functional materials ${ }^{1-3}$, especially composite systems composed of noble metal nanoparticles embedded in polymer matrixes. This is because of the result composite nanostructures have novel physical properties and potential applications.

Polymer nanofibers have been a subject of extensive research due to their unique properties. For the preparation of polymer nanofibers, the electrospinning method was thought to be a simple fiber fabrication technique and many polymer nanofibers have been prepared successfully. With electrospinning progress, it prove to be an interesting topic to introduce inorganic into polymer nanofiber in the process of electrospinning, such as inorganic oxide, inorganic quantum dot and noble metal nanoparticles have been electrospun into polymer nanofibers successfully ${ }^{4-10}$. Recently, Ag nanoparticle-embedded electrospun nanofibers, because of their applications in antimicrobial, have gained great attention. Yang et al. ${ }^{11}$ have succeeded in introducing Ag into poly(acrylonitrile) (PAN) nanofiber and then these hybrid systems of polymer fibers containning Ag compounds have been fabricated by electrospinning processes, such as Ag/PVP ${ }^{12}, \mathrm{Ag} / \mathrm{PVA}^{13}, \mathrm{Ag} / \mathrm{PVA} \mathrm{PVP}^{14}, \mathrm{Ag} /$ PCL-PU ${ }^{15}$, Ag/gelatin ${ }^{16}$ and $\mathrm{Ag} / \mathrm{CA}^{17}$.
It has been well known that $\mathrm{Ag}$ nanoparticles exhibit strong antibacterial properties. In this study, we fabricated $\beta$-cyclodextrin/poly(N-vinylpyrrolidone)/silver composite nanofibers by electrospinning technology and the prepared fiber mats were characterized by SEM and TEM. Antibacterial properties of the $\beta$-CD/PVP/silver composite nanofibers were investigated by Staphylococcus aureus and Escherichia coli as a model.

\section{EXPERIMENTAL}

Poly(vinylpyrrolidone) (PVP, AR, Mw = 1300000) was provided by Xiamen Sanland Chemicals Co. Limited. $\beta$ Cyclodextrin and silver nitrate $\left(\mathrm{AgNO}_{3}, 99.8 \%\right)$ was supplied by Sinopharm Chemical Reagent Co. Ltd. (Shanghai, P.R. China). Ethanol and N,N-dimethylformamide (DMF) were supplied by Beijing Chemicals Co. (China).

Synthesis of AgNPs/ $\beta$-CD-PVP composite solution: The poly(N-vinyl-pyrrolidone) solutions were prepared by dissolving poly(N-vinylpyrrolidone) (PVP) in ethanol and N,N-dimethylformamide (DMF) for $12 \mathrm{~h}$, the poly(N-vinyl-pyrrolidone) concentration of $8 \mathrm{wt} \%$ (w/w, with respect to $10 \mathrm{wt}$ \% PS), respectively. Then, $\beta$-cyclodextrin $(\beta-C D)$ was dropped into the 8 wt. \% PVP/DMF solution when the concentration of $\beta-\mathrm{CD}$ was $4.48 \mathrm{wt}$ \% , the reaction proceeded in room temperature for $12 \mathrm{~h}$. 
In order to prepare the composite solution containing $\mathrm{Ag}$ nanoparticles, $\mathrm{AgNO}_{3}$ was dissolved in the $\mathrm{PVP} /$ ethanol at $0{ }^{\circ} \mathrm{C}$ firstly and then the reaction mixture with rapidly stirred at $78{ }^{\circ} \mathrm{C}$ for $10 \mathrm{~min}$. After $c a .20 \mathrm{~min}$, the PVP solution containing $\mathrm{Ag}$ nanoparticles was mixed with $\beta$-CD-PVP/DMF solution. The concentrations of PVP and $\beta$-CD were 8 and 2 wt $\%$, respectively. The weight of $\mathrm{AgNO}_{3}$ was calculated on the basis of the molar ration of $\mathrm{Ag}$ and PVP monomer when the Ag/PVP was $1 / 10,1 / 15,1 / 20$, respectively.

Electrospinning $\boldsymbol{\beta}$-CD-PVP nanofibers containing AgNPs: The above solutions were subsequently loaded individually in a glass tube with a sharp nozzle (the inner diameter of was $0.6 \mathrm{~mm}$ ) and the copper wire was connected to a $15 \mathrm{kV}$ high-voltage electrical potential. An aluminum foil served as the counter electrode and the distance between the capillary and the substrate electrode was $15 \mathrm{~cm}$. The electrospinning was performed at room temperature.

The pure PVP and $\beta-C D / P V P$ nanofiber film was also prepared using the similar procedure as described above.

Characterization: UV-visible absorption spectra were measured at room temperature by Shimadzu UV-VIS-NIR scanning spectrophotometer (UV3150) with a variable wavelength between 300-900 nm using a $10 \mathrm{~mm}$ quartz cell. The morphologies and the diameter of the composite fibers were investigated by scanning electron microscope (SEM, S-3400N). The composite fibers were collected on carbon-coated copper grids and the morphologies of the Ag nanoparticles were observed with a JEM-2010 Transmission Electron Microscopy (TEM) operated at $200 \mathrm{KV}$.

Antibacterial testing: The antibacterial properties of AgNPs/ $\beta$-CD-PVP composite nanofibers against both grampositive Staphylococcus aureus (ACCC 01331) and gram- negative Escherichia coli (ACCC 11856) bacteria were tested according the modified Kirby-Bauer method.

Due to the water-solubility of PVP, the samples needed proceeding crosslinking reaction to enhance the water resistance of nanofiber membranes. The PVP, $\beta$-CD/PVP and AgNPs/ $\beta$-CD-PVP nanofiber webs were placed in oven at $150{ }^{\circ} \mathrm{C}$ for $3 \mathrm{~h}$ to complete crosslinking reaction. Then the treated membranes were cut into squares of $1 \mathrm{~cm}^{2}$.

Before putting the webs on the agar plates, the treated samples were disinfected by UV irradiation for $0.5 \mathrm{~h} 0.1 \mathrm{~mL}$ bacterial suspension ( $S$. aureus or E. coli) were removed in sterilized agar plate and spread uniformity. Then, one piece of AgNPs/ $\beta$-CD-PVP web was put in the agar plate having bacterial suspension and cultivated $36 \mathrm{~h}$ at $37^{\circ} \mathrm{C}$ in incubator. In the same manner, the PVP and $\beta$-CD/PVP webs were tested. The resulted zone of inhibition was observed.

\section{RESULTS AND DISCUSSION}

We prepared $\beta$-CD-PVP/Ag nanoparticle composite nanofibers. $\mathrm{AgNO}_{3}$ was directly reduced to $\mathrm{Ag}$ nanoparticles by ethanol in the PVP viscous solution and PVP protected the $\mathrm{Ag}$ nanoparticles from aggregation. Then, we added $\beta-\mathrm{CD}-$ PVP/DMF solution to Ag/PVP solution.

Fig. 1 shows UV-visible absorption spectra of aqueous solutions of PVP stabilized at different concentrations of Ag nanoparticles. The spectra exhibit an absorption band at around 410-420 nm, which is a typical plasmon band of Ag nanoparticles, suggesting the formation of Ag nanoparticles. This suggested that the Ag nanoparticles were existed in the solution.

Fig. 2 shows the SEM images of the as-prepared Ag/ $\beta$ CD-PVP. Smooth and long nanofibers have been prepared
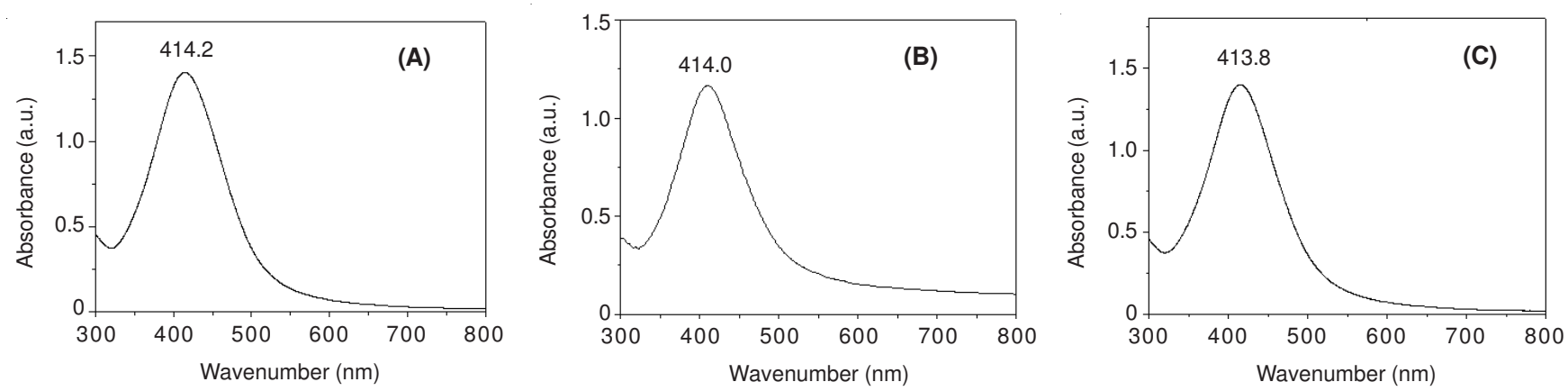

Fig. 1. UV-Visible absorption spectra of AgNPs/ $\beta$-CD-PVP composite solution at different concentrations of Ag nanoparticles: ((A) Ag/PVP $=1 / 10,(\mathrm{~B}) \mathrm{Ag} / \mathrm{PVP}=1 / 15$ and $(\mathrm{C}) \mathrm{Ag} / \mathrm{PVP}=1 / 20))$
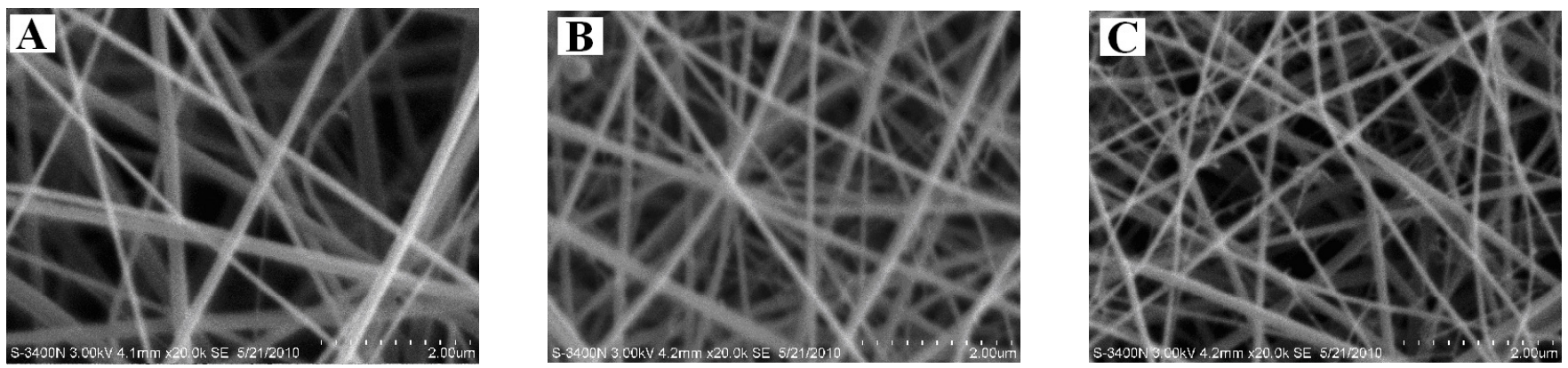

Fig. 2. SEM images of (A) Ag/ $\beta$-CD-PVP (Ag/PVP = 1/10) fibers, (B) Ag/ $\beta$-CD-PVP $(A g / P V P=1 / 15)$ fibers and $(C) A g / b-C D-P V P(A g /$ $\mathrm{PVP}=1 / 20$ ) fibers 
successfully by electrospinning. Fig. 2A-C showed that the diameters of Ag/ $\beta$-CD-PVP nanofibers obviously decreased with the increasing concentration of $\mathrm{Ag}$ nanoparticles in the composite solution. We know that the charge density increases with the addition of Ag nanoparticles in the solution, thus the ejected jets have stronger elongation force in the electrical field. Therefore, this resulted in forming thinner composite fibers $^{18}$.

Fig. 3 give the TEM images of the resulting $A g / \beta-C D-$ PVP composite nanofibers. Fig. 3A-C showed the different concentration of $\mathrm{Ag}$ nanoparticles in the solution, where the $\mathrm{PVP}$ and $\beta$-CD content in the solution is fixed in concentration ( 8 and $2 \mathrm{wt} \%$ ) in our experiments. When the content of $\mathrm{Ag}$ nanoparticles were $0.78,0.52$ and 0.39 wt $\%$ in the solution, the weight percentage of $\mathrm{Ag}$ nanoparticles in the obtained nanofiber composites were 7.8, 5.2 and $3.9 \mathrm{wt} \%$, respectively.
TEM images of composite nanofibers containing Ag nanoparticles, showed that Ag appeared as little dark spots inside of the nanofibers.

Fig. 4 shows the antimicrobial activity of the $\beta$-CD-PVP nanofibers containing Ag nanoparticles. Ag nanoparticles have the strong antimicrobial activity. In this study, the antimicrobial activity of the $\beta$-CD-PVP nanofibers containing Ag nanoparticles with a smaller average size can be confirmed by the clear zone of inhibition around the sample after $36 \mathrm{~h}$ incubation. The bacterial in the agar plate having PVP or $\beta-\mathrm{CD} /$ PVP web grow well, the sample have no influence on the growth of bacterial. In the agar plate with $\mathrm{Ag} / \beta-\mathrm{CD}-\mathrm{PVP}$, however, a clear zone of inhibition around the sample can be observed. The results of testing indicated that the composite nanofibers held antibacterial property against $S$. aureus and E. coli for the presence of silver nanoparticles.
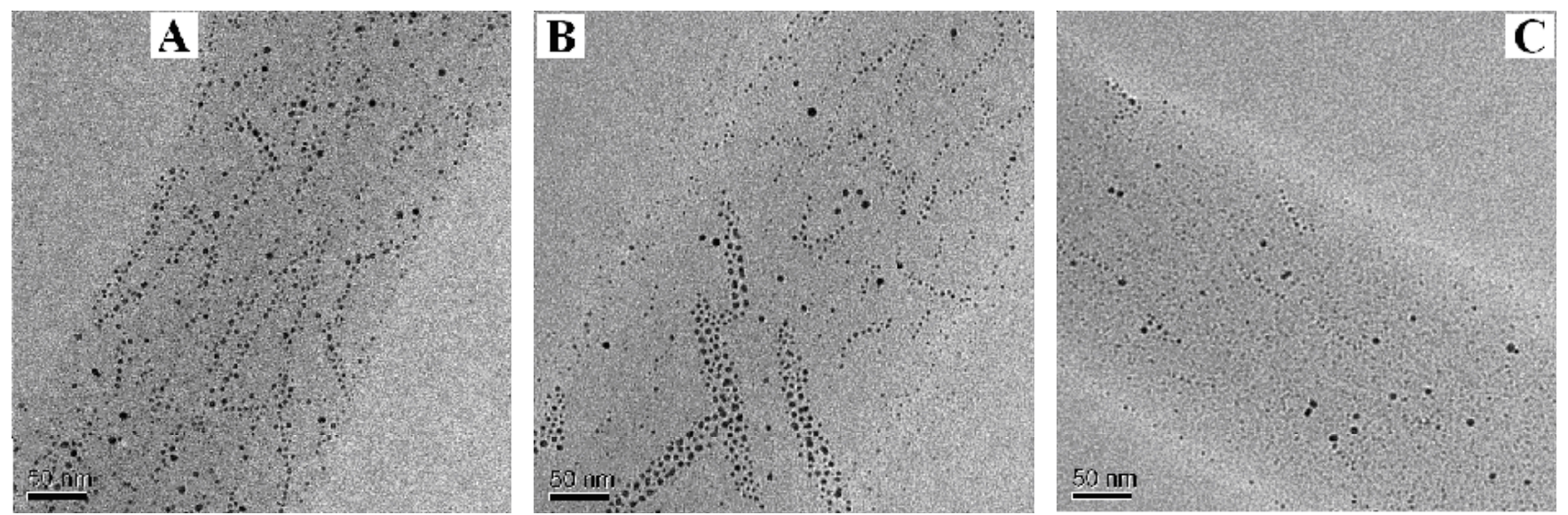

Fig. 3. TEM images of (A) Ag/b-CD-PVP (Ag/PVP = 1/10) fibers, (B) Ag/ $/$-CD-PVP (Ag/PVP = 1/15) fibers and (C) Ag/ $/ \mathrm{CD}-\mathrm{PVP}(\mathrm{Ag} /$ $\mathrm{PVP}=1 / 20)$ fibers
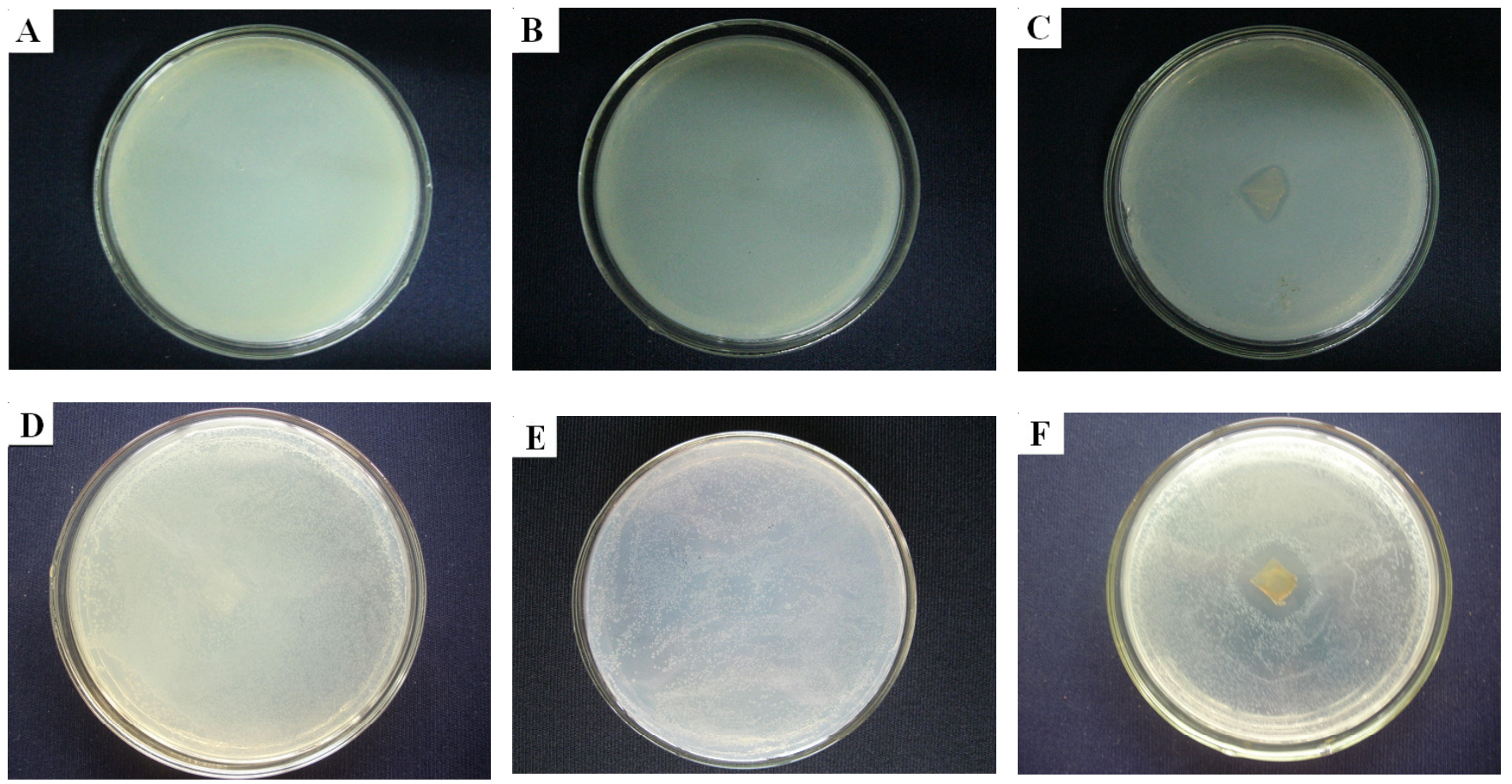

Fig. 4. Photograph images of the antibacterial test: (A) PVP nannofibers in plate with $S$. aureus, (B) $\beta$-CD/PVP nannofibers in plate with $S$. aureus, (C) Ag/ $\beta$-CD-PVP nannofibers in plate with $S$. aureus, (D) PVP nannofibers in plate with $E$. coli, (E) $\beta$-CD/PVP in plate with E. coli, (F) Ag/ $\beta-C D-P V P$ in plate with $E$. coli 


\section{Conclusion}

Hybrid nanofibers of $\beta$-CD/PVP with silver nanoparticles were successfully prepared by the electrospinning technique. The silver nanoparticles in the hybrid composites nanofibers were observed in their TEM images. Ag- $\beta$-CD/PVP nanofibers exhibited good antibacterial properties. This composite material may also have promising applications in antibacterial fields.

\section{REFERENCES}

1. B. Yang, K. Yoon and K. Chung, Mater. Chem. Phys., 83, 334 (2004).

2. A.L. Briseno, S.C.B. Mannsfeld, E. Formo, Y. Xiong, X. Lu, Z. Bao, S.A. Jenekhe and Y. Xia, J. Mater. Chem., 18, 5395 (2008).

3. M.K. Corbierre, N.S. Cameron and R.B. Lennox, Langmuir, 20, 2867 (2004).

4. X.M. Sui, C.L. Shao and Y.C. Liu, Appl. Phys. Lett., 87, 113 (2005).

5. L. Francis, F. Giunco, A. Balakrishnan and E. Marsano, Curr. Appl. Phys., 10, 1005 (2010).

6. X.F. Lu, Y.Y. Zhao and C. Wang, Adv. Mater., 17, 2485 (2005).
7. B. Maddah, M. Azimi, S. Imani, A.M. Zand, H. Borna and M. Sharifi, Asian J. Chem., 25, 217 (2013).

8. K. Cho, M. Kim, J. Choi, K. Kim and S. Kim, Synth. Met., 160, 888 (2010).

9. G. Han, B. Guo, L. Zhang and B. Yang, Adv. Mater., 18, 1709 (2006).

10. M.M. Demir, M.A. Gulgun and Y.Z. Menceloglu, Macromolecules, 37, 1787 (2004).

11. Q.B. Yang, D.M. Li , Y.L. Hong, Z.Y. Li, C. Wang, S.L. Qiu and Y. Wei, Synth. Met., 137, 973 (2003).

12. W. Jin, H.K. Lee, E.H. Jeong, W.H. Park and J.H. Youk, Macromol. Rapid Commun., 26, 1903 (2005).

13. W.J. Jin, H.J. Jeon, J.H. Kim and J.H. Youk, Synth. Met., 157, 454 (2007).

14. G.P. Dong, X.D. Xiao, X.F. Liu, B. Qian, Z.J. Ma, S. Ye, D.P. Chen and J.R Qiu, J. Nanopart. Res., 12, 1319 (2010).

15. H.J. Jeon, J.S. Kim, T.G. Kim, J.H. Kim, W.R. Yu and J.H. Youk, Appl. Surf. Sci., 254, 5886 (2008).

16. P. Rujitanaroj, N. Pimpha and P. Supaphol, Polymer, 49, 4723 (2008).

17. W.K. Son, J.H. Youk and W.H. Park, Carbohydr. Polym., 65, 430 (2006).

18. J. Bai, Y.X. Li, S.T. Yang, J.S. Du, S.G. Wang, J.F. Zheng, Y.Z. Wang, Q.B. Yang, X.S. Chen and X.B. Jing, Solid State Commun., 141, 292 (2007). 